\title{
Reaction of REBaCuO CC Tapes to Neutron Irradiation
}

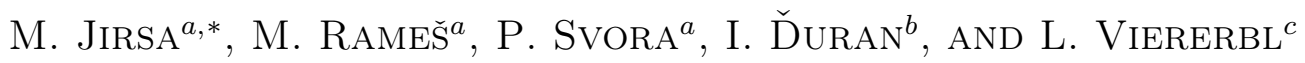 \\ ${ }^{a}$ Institute of Physics CAS, Na Slovance 2, CZ-18221 Prague 8, Czech Republic \\ ${ }^{b}$ Institute of Plasma Physics CAS, Za Slovankou 1782/3, CZ-18200 Prague 8, Czech Republic \\ ${ }^{c}$ Research Centre Rež, Hlavní 130, CZ-25068 Husinec-Řež, Czech Republic
}

\begin{abstract}
Superconducting $\mathrm{REBaCuO}(\mathrm{RE}=\mathrm{Gd}, \mathrm{Y}+\mathrm{Gd})$ tapes intended for wiring magnets for fusion reactors were investigated in response to neutron irradiation by fast neutron fluences up to $4 \times 10^{22} \mathrm{~m}^{-2}$. The results indicate that the tapes are appropriate for the given purpose, in particular at temperatures below $25 \mathrm{~K}$. There, due to neutron irradiation, the critical currents at low magnetic fields are gradually reduced, while in high magnetic fields they are first enhanced, then degraded. The position of the crossover differs from one tape type to another, in dependence on the initial pinning landscape. To find reasons for such behavior, SEM and TEM analyses started. Some preliminary SEM results are presented. The effect of irradiation on pinning landscape is discussed.
\end{abstract}

DOI: 10.12693/APhysPolA.137.779

PACS/topics: superconducting tapes, critical currents, neutron irradiation, fusion reactor magnets

\section{Introduction}

In pursuit of developing a powerful and clean source of energy for future demands, fusion reactors as a source of energy have received a great interest as one of the best candidates. Fusion reactor is based on plasma confined in a relatively small space by a mighty magnetic field. Currently, only superconducting magnets are capable of delivering magnetic field high enough. Now constructed and designed fusion reactors, like DEMO, ITER, CFETR etc., rely on well-established classical superconductors, cooled by liquid helium [1]. In parallel, attempts are being made to use high temperature magnets, e.g., [2-5]. Although high- $T_{c}$ superconductors exhibit satisfactory properties only at temperatures below $25 \mathrm{~K}$ [6, 7], they are good for use at high magnetic fields, while compared to conventional counterpart they possess great advantages in wide safety margins, both in temperature and magnetic field. One of the important issues to be understood is the tapes' response to neutron irradiation, which is always present in the fusion reactors. In dependence on the neutron energy and the superconductor type, the irradiation produces in superconductor point-like defects or their cascades [8-10]. In most cases the pinning defect structure densifies, which leads to vortex pinning increase and the associated critical current enhancement. The process, however, depends on the initial pinning structure. The increasing neutron fluence finally brings the structure to the stage when vortex lattice does not further react on the defect densification, and the electromagnetic properties start degrading. This process depends on temperature, magnetic field, superconductor type, and neutron fluence.

*corresponding author; e-mail: jirsa@fzu.cz
In this paper, we present some data observed on tapes of various producers, and thus complementing the complex behavioral map of the superconducting tape in response to neutron irradiation.

\section{Experimental details}

The tapes studied in this paper, i.e., Gd-123 (SuperOx) and $(\mathrm{Y}+\mathrm{Gd})-123$ (SuperPower) are superconducting tapes of the second generation $(2 \mathrm{G})$ prepared on Hastelloy substrates and protected by a copper sheaths. The width of the tapes $w$ was $4 \mathrm{~mm}$, and the total thickness ranged between 0.1 and $0.3 \mathrm{~mm}$. The superconducting layer was about $1 \mu \mathrm{m}$ thick. The tapes were first measured before irradiation [11], and next after each irradiation step. Electromagnetic properties were evaluated in terms of critical currents $I_{c}$ calculated from magnetic hysteresis loops (MHL) measured by means of a vibrating sample magnetometer (VSM). For a rectangular sample one used the Bean model formula $[12,13]$

$$
I_{c}=w c J_{c}=\frac{20 \Delta m}{a^{2} b c\left(1-\frac{a}{3 b}\right)},
$$

where $w=4 \mathrm{~mm}$ is the tape width, $c$ is the superconducting layer thickness, $J_{c}$ is critical current density, sample dimensions $a$ and $b$ are transversal to magnetic field direction, such that $b \geq a$, and $\Delta m$ is the MHL height. For the inductive measurements samples were cut by an electrical discharge of wire-cut machine in dimensions $a \times b=1.5 \times 1.5 \mathrm{~mm}^{2}$. In most cases the tests were done at temperatures: 10, 50, and $77 \mathrm{~K}$. When $a, b$ and $w$ are given in $\mathrm{mm}, c$ in $\mu \mathrm{m}$, and $\Delta m$ in memu (i.e., $10^{-6} \mathrm{~A} \mathrm{~m}^{2}$ ), then the $J_{c}$ goes as $10^{6} \mathrm{~A} / \mathrm{cm}^{2}$. The VSM magnetometer is an option of the Physical Property Measuring system with 9 T magnet (PPMS 9).

To avoid the problem of, not always exactly known, superconductor thickness $c$, we use the $I_{c}$ representation. From (1) one can deduced $I_{c}$ and it should be equivalent to the transport currents measured on the same tapes 

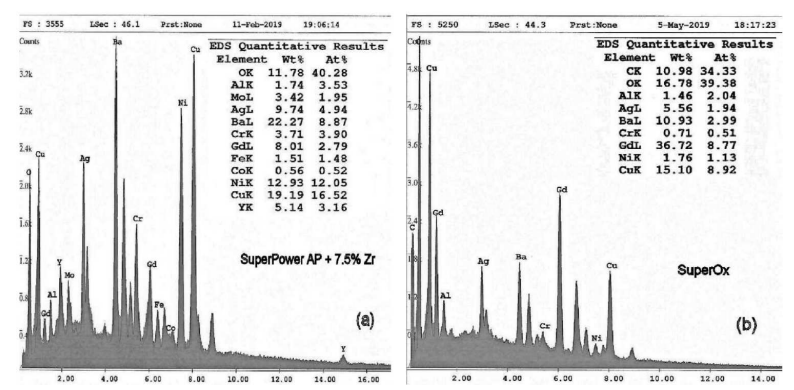

Fig. 1. EDX spectra measured on the superconducting layers of the tapes.

(4 mm wide). However, in reality these two currents need not be exactly identical because they are measured at different relaxation states and on significantly different sample sizes. In all induction measurements magnetic field was applied parallel to $c$-axis.

The neutron irradiation was made with total neutron fluences of $2.1 \times 10^{22} \mathrm{~m}^{-2}, 1 \times 10^{22} \mathrm{~m}^{-2}$, and $8.03 \times 10^{22} \mathrm{~m}^{-2}$ in the LVR-15 research reactor [14]. Selected samples were fixed, each in one of the bores drilled into an Al block-holder. This holder was placed into a glass ampule wrapped by $\mathrm{Cd}$ foil to shield the samples from thermal neutrons (with energies $0-0.55 \mathrm{eV}$ ). For the highest fluence step, the fluence of thermal neutrons was $6.08 \times 10^{20} \mathrm{~m}^{-2}$, while fluence of the part with intermediate energies $(0.55 \mathrm{eV}-0.11 \mathrm{MeV})$ was $5.07 \times 10^{22} \mathrm{~m}^{-2}$. The fluence of fast neutrons $(E \geq 0.1 \mathrm{MeV})$ was $2.9 \times 10^{22} \mathrm{~m}^{-2}$, which represented cca $36 \%$ of the total fluence. Fluences were measured with three sets of activation detectors fixed near the irradiated samples, inside the $\mathrm{Cd}$ foil. Each set contained four detectors based on $\mathrm{Ti}, \mathrm{Fe}, \mathrm{Ni}$ and Co foils. Induced activities were measured with the HPGe detector, while fluences were evaluated by the STAYSL code [15]. The ampule was cooled to $55^{\circ} \mathrm{C}$ during irradiation, however, temperature inside the sample might be even higher, i.e., between $70^{\circ} \mathrm{C}$ and $130^{\circ} \mathrm{C}$ (guess) due to further radiation heating. In this point one can see that the irradiation conditions differ from reality in future fusion reactors. The sample irradiation at low temperatures requires a substantial reconstruction of the facility, which is still under consideration.

Some basic information on the structure and composition of the tapes was achieved by scanning electron microscopy (SEM) by means of SEM-FEI Phenom $(5 \mathrm{kV}$, $24 \mathrm{k}$ magnification, $60 \mathrm{~nm}$ resolution) BSE detector (material contrast), SEM/FIB-FEI QUANTA 3S FEG with EDAX EDX. Some results on the tapes composition are shown in Fig. 1.

\section{Experimental results}

Results concerning the superconductor composition are shown in Fig. 1. The main result of the EDX analysis is that the SuperPower $\mathrm{AP}+7.5 \% \mathrm{Zr}$ tape is $\approx\left(\mathrm{Y}_{0.5} \mathrm{Gd}_{0.5}\right) \mathrm{BaCuO}$, while the SuperOx tape is

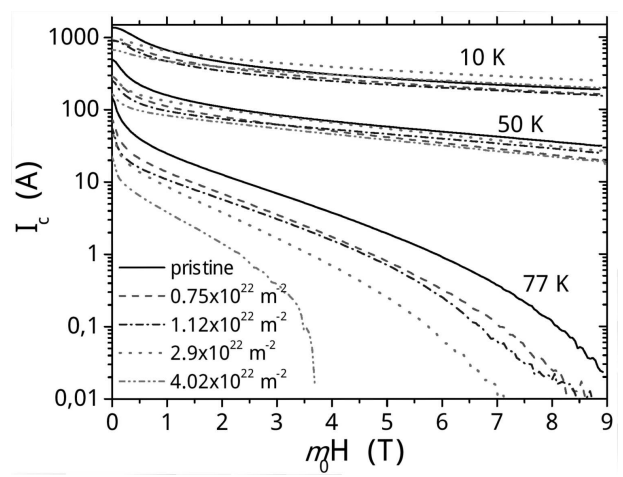

Fig. 2. Development of the field dependence of $I_{c}$ on the fast neutron fluence for a SuperOx tape.
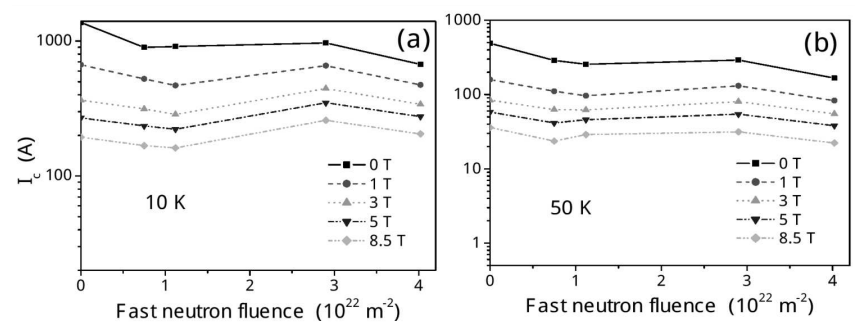

Fig. 3. Development of $I_{c}$ the fast neutron fluence at $10 \mathrm{~K}(\mathrm{a})$, and $50 \mathrm{~K}$ (b) for a SuperOx tape.

$\mathrm{GdBaCuO}$ with rather high content of Gd and surprisingly low amount of Ba. The declination of the composition from the stoichiometric one in the latter tape might be due to a rather high content of 211 secondary phase and $\mathrm{Gd}_{2} \mathrm{O}_{3}$ particles added usually as vortex lattice pins. Both tapes are nearly equally oxygenated. To our surprise, no zirconium was detected in the SuperPower tape. A more precise information is expected from TEM.

The development of $I_{c}$ field dependence with increasing fast neutron fluence is displayed in Fig. 2 for SuperOx tape. One can see that irradiation effect differs at different temperatures and magnetic fields.

In order to get more precise insight into $I_{c}$ dependence on neutron fluence, the data for $10 \mathrm{~K}$ were transformed and presented in Fig. 3 as $I_{c}$ dependence on the fluence of fast neutrons for various magnetic fields (Fig. 3a). The same was done for $50 \mathrm{~K}$ in Fig. 3b. It is evident that at various magnetic fields the response was different.

We have normalized the data from Fig. 3 by the $I_{c}$ values of the intact tape (zero neutron fluence) to be able to quantify the $I_{c}$ enhancement/degradation. The results are shown in Figs. $4 \mathrm{a}$ and $4 \mathrm{~b}$, for $10 \mathrm{~K}$ and $50 \mathrm{~K}$, respectively.

Figure 4a shows that at low temperatures, the neutron irradiation causes degradation of $I_{c}$ values in all investigated fields. Then, the $I_{c}(\varphi)$ curves turn up, and those for high magnetic fields rise above the value for the intact tape, reaching maximum and again falling gradually below the "intact" value. In opposite, at s.f. (0 T), 

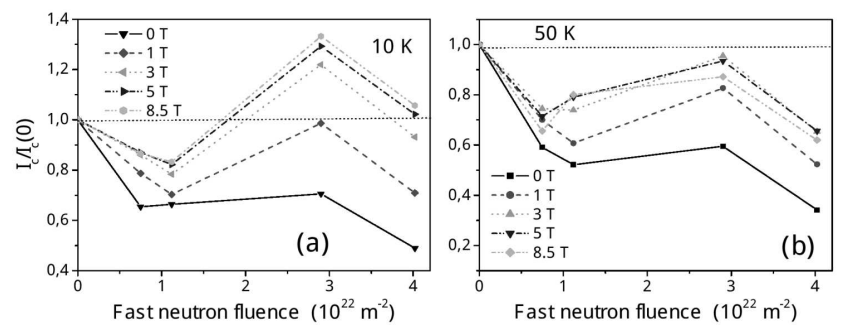

Fig. 4. The relative enhancement and/or degradation of $I_{c}$ due to neutron irradiation with respect to the value of the intact tape at $10 \mathrm{~K}$ (a), and (b) $50 \mathrm{~K}$ for a SuperOx tape.

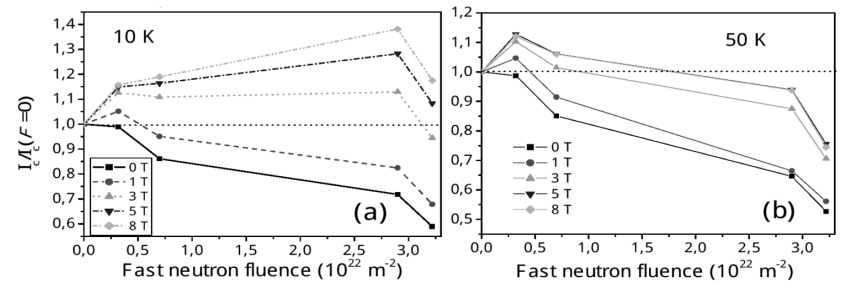

Fig. 5. The relative enhancement and/or degradation of $I_{c}$ due to neutron irradiation with respect to the $I_{c}$ value of the intact tape at $10 \mathrm{~K}(\mathrm{a})$, and (b) $50 \mathrm{~K}$, for a SuperPower tape 4050 AP with $7.5 \% \mathrm{Zr}$.

$I_{c}$ degrades and then stays nearly constant over a wide range of neutron fluences (the lowest curve in Fig. 4a). Such a behavior is qualitatively in accord with the experiments done by current transport [16]. At intermediate and high temperatures (Fig. 4b), the $I_{c}(\varphi)$ dependence behaves similar to that at low temperatures (Fig. 4a), however the cross-over at the intermediate irradiation fluence does not reach the intact tape level for any magnetic field. At temperatures above $70 \mathrm{~K}$, relaxation support through intense thermal activation [7] is so high that $I_{c}$ degrades with both growing neutron fluence and increasing magnetic field. A similar behavior, at least qualitatively, is quite general, as shown in Fig. 5 on the SuperPower tape 4050 AP doped by $7.5 \% \mathrm{Zr}$. There are small differences caused by a different initial pinning landscape of the two superconductors. Increasing temperature shifts the turn point of the $I_{c}(\varphi) / I_{c}(0)$ curves to lower neutron fluences. TEM investigation is just underway, which will provide a detail information on the tapes' microstructure.

\section{Conclusion}

Effect of neutron irradiation was studied on a series of commercial $2 \mathrm{G}$ superconducting tapes. Results of two of them, that come from different manufacturers, indicate that the irradiation effect is quite general, despite of the tape type and the experimental method employed. At temperatures below $30 \mathrm{~K}$, the values of $I_{c}$ at high magnetic fields grows with the irradiation fluence but at a certain value this tendency changes into a gradual degradation of $I_{c}$. However, at about $4 \times 10^{22} \mathrm{~m}^{-2}$, $I_{c}$ is still close to the value of intact tape, which is a good message for potential use of these tapes in fusion reactors as this fluence is above the total life-time value in most reactors.

\section{Acknowledgments}

This work was carried out within the framework of the EUROfusion Consortium and received funding from the Euratom research and training program 2014-2018 under grant agreement No. 633053. The views and opinions expressed herein do not necessarily reflect those of the European Commission. Experiments were performed in the Materials Growth and Measurement Laboratory MGML (http://mgml.eu). Irradiation costs were covered by the MEYS project LM2015074.

\section{References}

[1] N. Mitchell, A. Devred, P. Libeyre, B. Lim, F. Savary, IEEE Trans. Appl. Supercond 22, 4200809 (2012).

[2] P. Bruzzone, W.H. Fietz, J.V. Minervini, M. Novikov, N. Yanagi, Y. Zhai, J. Zheng, Nucl. Fusion 58, 103001 (13 pp) (2018).

[3] K. Ding, Y. Bi, H. Feng et al., Phys. Procedia 36, 931 (2012).

[4] R. Wesche, X. Sarasola, K. Sedlak, N. Bykovsky, B. Stepanov, D. Uglietti, P. Bruzzone, IEEE Trans. Appl. Supercond. 28, 4203605 (2018).

[5] D. Uglietti, N. Bykovsky, K. Sedlak, B. Stepanov, R. Wesche, P. Bruzzone, Supercond. Sci. Technol. 28, 124005 (2015).

[6] R. Prokopec, D.X. Fischer, H.W. Weber, M. Eisterer, Supercond. Sci. Technol. 28, 014005 (2015).

[7] M. Jirsa, M. Rameš, I. Ďuran, T. Entler, L. Viererbl, Supercond. Sci. Technol. 32, 055007 (2019).

[8] M. Eisterer, R. Fuger, M. Chudy, F. Hengstberger, H.W. Weber, Supercond. Sci. Technol. 23, 014009 (2010).

[9] M. Chudy, R. Fuger, M. Eisterer, H.W. Weber, IEEE Trans. Appl. Supercond. 21, 3162 (2011).

[10] J. Emhofer, M. Eisterer, H.W. Weber, Supercond. Sci. Technol. 26, 035009 (2013).

[11] M. Jirsa, M. Rameš, I. Ďuran, T. Melíšek, P. Kováč, L. Viererbl, Supercond. Sci. Technol. 30, 045010 (2017).

[12] C.P. Bean, Rev. Mod. Phys. 36, 31 (1964).

[13] D.X. Chen, R.B. Goldfarb, J. Appl. Phys. 66, 2489 (1989).

[14] M. Koleška, Z. Lahodová, J. Šoltés, L. Viererbl, J. Ernest, M. Vinš, J. Stehno, J. Radioanal. Nucl. Chem 305, 51 (2015).

[15] ASTM E944-08, Standard Guide for Application of Neutron Spectrum Adjustment Methods in Reactor Surveillance, E706 (IIA), ASTM International, West Conshohocken (PA) 2008.

[16] D.X. Fischer, R. Prokopec, J. Emhofer, M. Eisterer, Supercond. Sci. Technol. 31, 044006 (2018). 